\title{
OUTCOME AFTER AZACITIDINE TREATMENT IN PATIENTS WITH HIGH-RISK MYELODYSPLASTIC SYNDROME AND ACUTE MYELOID LEUKEMIA IN THE CLINIC OF HEMATOLOGY AT ST. MARINA UNIVERSITY HOSPITAL, VARNA
}

\author{
Ilina Micheva ${ }^{1,2}$, Vladimir Gerov ${ }^{1,2}$, Stela Dimitrova ${ }^{1,2}$, Merlin Efraim $^{1,2}$, Liana Gercheva ${ }^{1,2}$ \\ ${ }^{1}$ Department of Internal Diseases, Faculty of Medicine, Medical University of Varna \\ ${ }^{2}$ Clinic of Hematology, St. Marina University Hospital, Varna
}

\begin{abstract}
INTRODUCTION: Hypomethylating agents have become a standard therapy for high-risk myelodysplastic syndromes (MDS) and elderly patients with acute myeloid leukemia (AML).

AIM: The aim of the study was to assess the efficacy of azacitidine treatment in patients with MDS and AML followed for 18 months.

MATERIALS AND METHODS: Twenty-seven patients with MDS and AML treated in the Clinic of Hematology at St. Marina University Hospital, Varna were included in the study. Azacitidine was administered subcutaneously at a dose of $75 \mathrm{mg} / \mathrm{m}^{2}$ for 7 days. Disease assessment was performed on the $3^{\text {rd }}$ month, $6^{\text {th }}$ month, and at progression.

RESULTS: Twenty-seven patients were analyzed. Their median age was 71.5 years. Nine had refractory anemia with excess of blasts II (RAEB II), 5 had chronic myelomonocytic leukemia II (CMML II), 1 was with unclassifiable MDS (MDS-U), and 12 with AML. The median number of administered cycles was 6 (1-19). Eleven patients completed 6 cycles of azacitidine. Partial response was achieved in 9 patients (33\%) (7 MDS and 2 AML), stable disease in 8 (29\%) (5 MDS and 3 AML). Progressive disease was observed in 10 patients (37\%). The response correlated with the type of the disease $(\mathrm{p}=0.03)$, cytogenetic risk $(\mathrm{p}=0.01)$, and survival $(p=0.000)$. At 18 months, $60 \%$ of MDS patients were alive compared to $41.7 \%$ in the AML group. The median time to death in the AML patient group was 2.5 months. The mean overall survival was 10.4 months (12.6 months for MDS patients and 5.4 months for AML patients).

CONCLUSION: The therapy with azacitidine is an option for elderly patients with high-risk MDS. In patients with AML a rapid progression is observed during the first two cycles with mortality rate of $58.3 \%$. Scr Sci Med. 2018;50(1):31-35
\end{abstract}

Address for correspondence:

Ilina Micheva

Keywords: $M D S, A M L$, hypomethylating agents,

Clinic of Hematology

St. Marina University Hospital

1 Hr. Smirnenski Blvd

9000 Varna

e-mail: ilinamicheva@gmail.com azacitidine

Received: February 18, 2018

Accepted: March 26, 2018 


\section{INTRODUCTION}

DNA methylation is an important mechanism of epigenetic gene regulation. In human cells, DNA methylation occurs in cytosine residues that precede guanines $(\mathrm{CpG})$ grouped in $\mathrm{CpG}$-rich regions known as $\mathrm{CpG}$ islands. These islands are usually not methylated in normal cells. The methylation status of DNA is controlled by a family of DNA methyltransferases (DNMTs) (1). It is well known that complex changes in DNA methylation are characteristic feature of many cancers; tumor suppressor genes can be silenced by aberrant hypermethylation (2). Hypermethylation is found in patients with myeloid malignancies affecting hundreds of genes, involved in different signaling pathways. It has been shown that aberrant hypermethylation of genes is widespread in patients with myelodysplastic syndromes (MDS) and secondary acute myeloid leukemia (AML) (3). Hypermethylation increases with advanced disease and is a poor prognostic factor in MDS and AML. Patients with hypermethylation of at least one tumor suppressor gene have shorter survival (4).

This underlies the clinical efficacy of the hypomethylating agents for the treatment of patients with MDS and AML. Azacitidine is an analog of cytosine, where the carbon is replaced by nitrogen at the 5-position on the cytosine ring. DNMTs bind irreversibly to the cytosine analog but are unable to methylate it, which causes depletion of cellular DNMT levels. Azacitidine is integrated into both RNA and DNA, causing DNA hypomethylation and cytotoxicity with DNA damage and inhibition of protein synthesis (5).

Hypomethylating agents have become a standard therapy for high-risk MDS, chronic myelomonocytic leukemia II (CMML II) and elderly patients with AML who are not eligible for hematopoietic stem cell transplantation.

\section{AIM}

The aim of the study was to assess the efficacy of azacitidine treatment in higher risk patients with MDS and AML. The clinical workup included response assessment and survival.

\section{MATERIALS AND METHODS}

A group of 27 patients, 15 men and 12 women, with MDS and AML, treated in the Clinic of
Hematology, St. Marina University Hospital, Varna were included in the study. Patients were eligible for azacitidine treatment if they were with highrisk MDS, determined by the revised international prognostic scoring system (IPSS-R) (6), CMML II and AML, aged 65 years or older, not eligible for intensive chemotherapy and hematopoietic stem cell transplantation. Azacitidine was administered subcutaneously in a standard dose of $75 \mathrm{mg} / \mathrm{m}^{2}$ per day for 7 days, every 28 days. Blood counts were done monthly. Disease assessment was performed on the $3^{\text {rd }}$ and the $6^{\text {th }}$ month after initiation of the treatment, or at progression. Response was evaluated according to the International Working Group (IWG) response criteria in myelodysplasia (7). Mean survival was estimated by the Kaplan-Meier method. For evaluation of linear relationships, a two-tailed Pearson Correlation test was used. Correlation was considered significant at the 0.05 level. Statistical analysis was performed using SPSS 19.

\section{RESULTS}

Between June 2016 and December 2017, 27 patients were treated with azacitidine. According to the WHO classification 9 patients were with refractory anemia with excess of blasts II (RAEB II), 5 with CMML II, 1 with MDS unclassifiable (MDS-U) and 12 with AML. Median age was 71.5 years (range 4480 ) and $70.3 \%$ of the patients were over 65 years. According to IPSS-R 2 MDS patients were scored as very high risk, 1 as high, and 5 as intermediate (Fig. 1a). Four of the AML patients were high and 8 - intermediate risk (Fig.1b). According to cytogenetics 16 patients were with normal karyotype, 6 with complex, and 5 patients were with other cytogenetic abnormalities [46, XY, del (7) $(\mathrm{n}=2), 46, \mathrm{XY}$, add(16q) $(\mathrm{n}=1), 47, \mathrm{XY},+19(\mathrm{n}=1), 47, \mathrm{XY},+\mathrm{C}(\mathrm{n}=1)]$. In 23 patients the treatment with azacitidine was first-line chemotherapy. In 3 patients with AML, azacitidine was initiated after failure of $7+3$ or low doses Ara-C, 1 patient with CMMLII was previously treated with hydroxyurea.

Azacitidine was given for a median of six cycles (1-19). The median azacitidine cycle length was 28 days. Seventeen patients completed three cycles of treatment, 11 patients - six cycles. Disease assessment was performed on the $3^{\text {rd }}$ and the $6^{\text {th }}$ month after ini- 
Ilina Micheva, Vladimir Gerov, Stela Dimitrova et al.
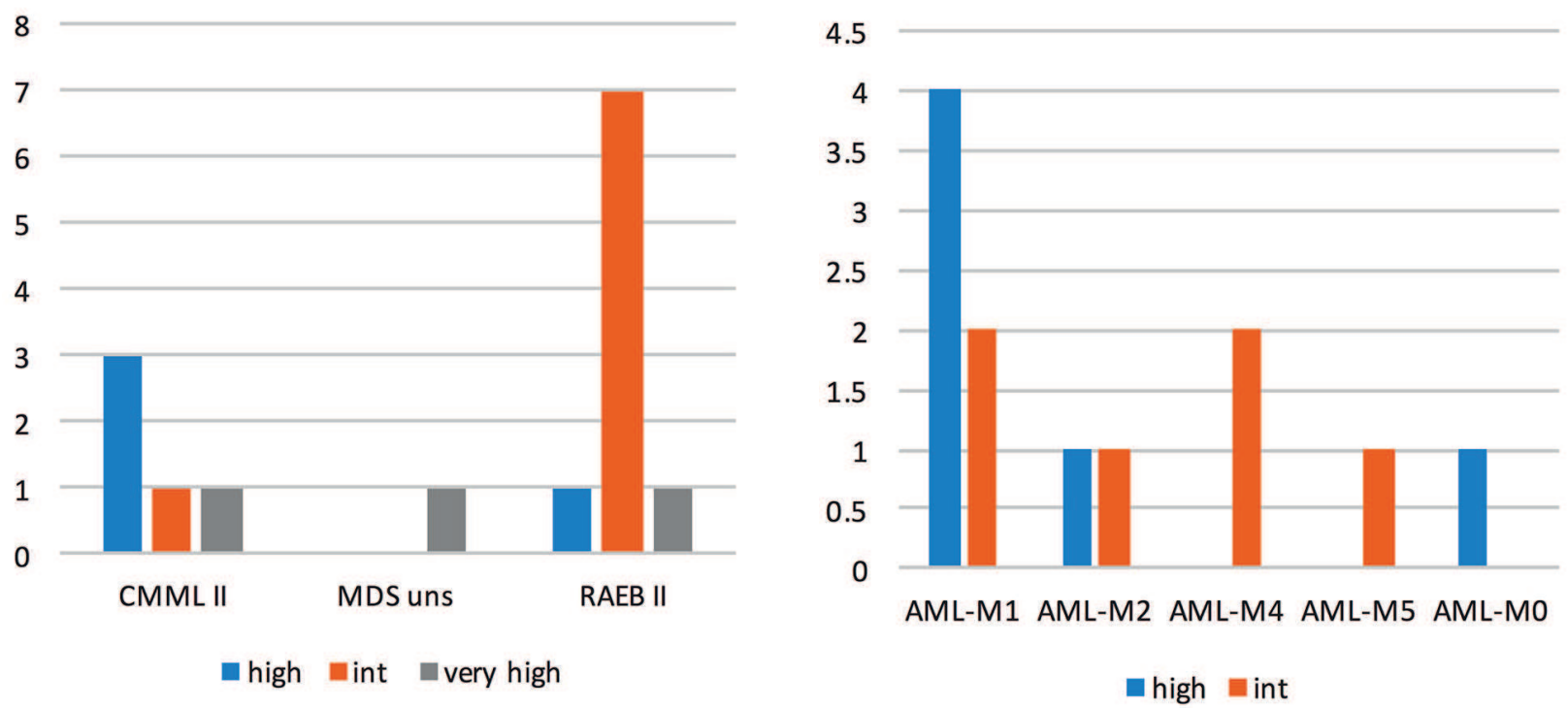

Fig. 1. Risk stratification in MDS (a) and AML patients (b)

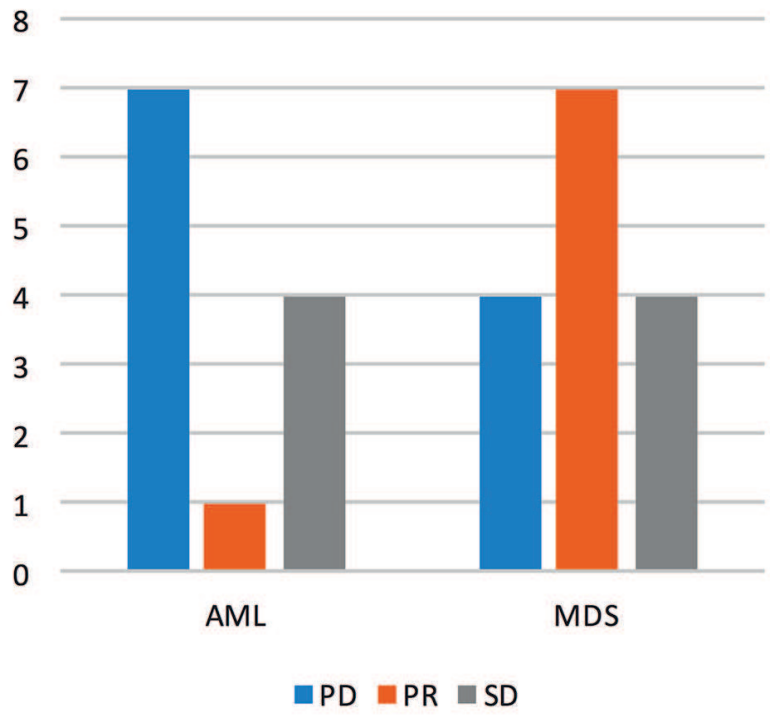

Fig. 2. Response achieved during the treatment in MDS and AML patients

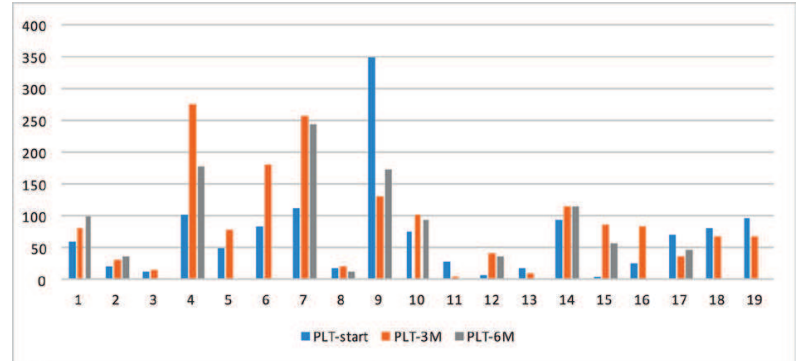

tiation of the treatment, or at progression. The best response is presented on Fig. 2.

Partial response (PR) was achieved in 9 patients (33\%) (7 with MDS and 2 with AML). Eight patients (29\%) had stable disease (SD) (5 with MDS and 3 with AML). Ten patients (37\%) had progressive disease (PD). The response correlated with the type of the disease (MDS or AML) ( $\mathrm{p}=0.03)$, cytogenetic risk $(\mathrm{p}=0.01)$ and survival $(\mathrm{p}=0.000)$. AML transformation was observed in 3 patients with MDS (MDS-U, CMML II, and RAEB II) with time of transformation 3, 6, and 15 months, respectively. Hematological improvement (HI) of hemoglobin was achieved in 5 patients and $\mathrm{HI}$ of platelets - in 9 patients (Fig. 3).

All patients with $\mathrm{HI}$ became transfusion-independent. The age had no influence on response and survival. At 18 months, on the basis of Kaplan-Meier estimates, $60 \%$ of all MDS patients were alive compared with $41.7 \%$ in the AML group. All patients

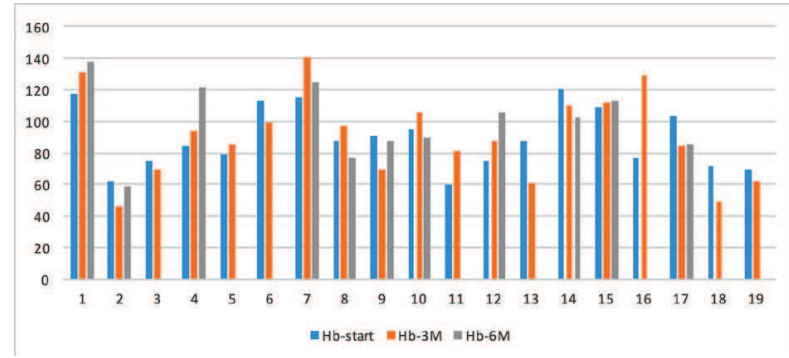

Fig. 3. Levels of hemoglobin (a) and platelets (b) measured prior to treatment, and on the $3^{\text {rd }}$ and the $6^{\text {th }}$ month 
died as a result of PD with a median time to death 2.5 months. Mean Kaplan-Meier overall survival was 10.4 months: 12.6 months for MDS patients and 5.4 months for AML patients (Fig. 4).

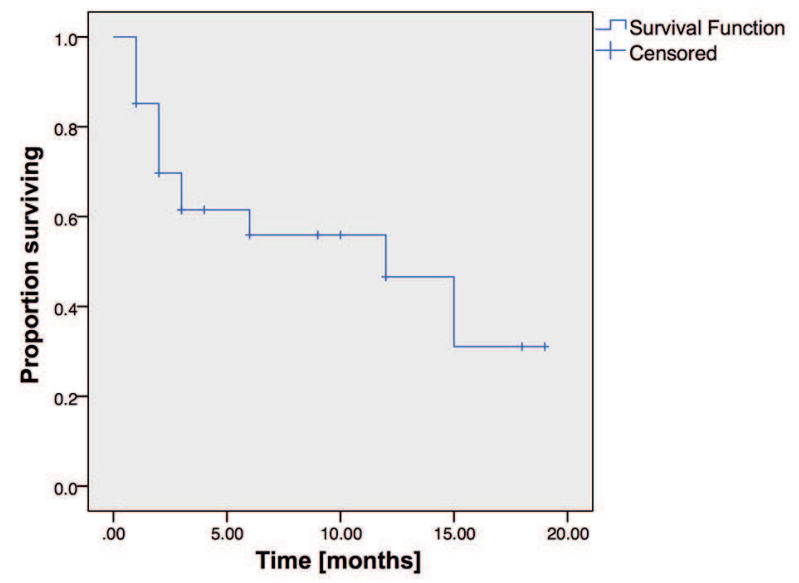

able karyotypes, poor performance status, and comorbidities. As a result, many older patients receive only palliative care. There is no universally accepted standard approach to treating AML in older patients.

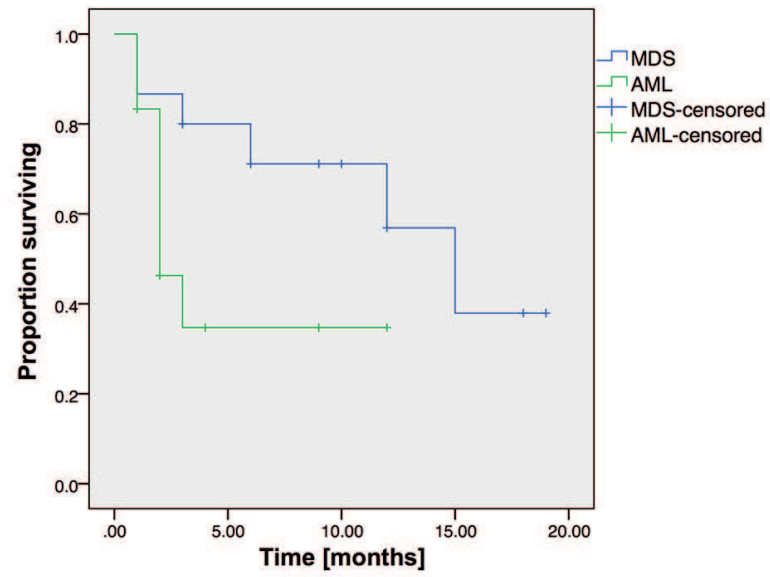

Fig. 4. Overall survival (a). Overall survival in MDS and AML (b)

\section{DISCUSSION}

Myelodysplastic syndromes comprise a heterogeneous group of hematopoietic stem cell disorders that differ by clinical phenotype and risk stratification (8). Patients with high-risk MDS have inferior survival and a high-risk for progression to AML. The median overall survival in very high and highrisk MDS patients is 0.8 and 1.6 years, respectively (6). The therapeutic options in these risk groups are limited; no treatment strategies other than allogeneic stem-cell transplantation offer the potential to change the natural history of the disease (9). The first study of a Cancer and Leukemia Group B, comparing treatment with azacitidine with best supportive care in high-risk MDS patients suggested improved overall survival with azacitidine (10). These results were confirmed by a large, prospective, randomized, phase III, clinical trial in which the median overall survival was 24.5 months for the azacitidine group versus 15.0 months for the conventional care group (11).

AML is an aggressive malignancy with poor prognosis. Older patients with AML over 65 years of age, who are at greatest risk of developing AML, have particularly poor survival rates (12). Treatment options are limited because of a variety of adverse prognostic risk factors, such as history of MDS, unfavor-
It was recently shown that azacitidine increases median overall survival by 3.8 months compared to current commonly used AML treatments (13).

In our study, after 18 months of follow-up, $60 \%$ of high-risk MDS patients were alive and continued their treatment. $46.6 \%$ of MDS patients have achieved PR and $26.6 \%$ remained in SD. Three patients died because of transformation into AML. Median survival is 12.6 months. In the AML patient group, $58.3 \%$ died within a median time of 2.5 months. However, 5 out of the 12 patients with AML continued their treatment at the end of the 18-month follow-up - 2 with PR and 3 with SD. All patients with MDS and AML who achieved PR or SD showed improvement or stabilization of blood counts with reduction of the number of infectious complications and transfusions during azacitidine treatment. Patients with MDS achieved better response compared to AML and showed better survival rates.

\section{CONCLUSION}

The therapy with azacitidine is an option for elderly patients with high-risk MDS. Patients with AML show rapid progression during the first two cycles of azacitidine with high mortality. This is the first study reporting results form azacitidine treat- 
Ilina Micheva, Vladimir Gerov, Stela Dimitrova et al.

ment in Bulgaria. A larger cohort of patients and longer follow-up is required for further conclusions.

\section{REFERENCES}

1. Weber M, Hellmann I, Stadler MB, Ramos L, Pääbo S, Rebhan M, et al. Distribution, silencing potential and evo- lutionary impact of promoter DNA meth- ylation in the human genome. Nat Genet. 2007;39(4):457-66. doi: 10.1038/ng1990.

2. Esteller M. Epigenetics in cancer. N Engl J Med. 2008;358(11):1148-59. doi: 10.1056/NEJMra072067.

3. Figueroa ME, Skrabanek L, Li Y, Jiemjit A, Fandy TE, Paietta E, et al. MDS and secondary AML display unique patterns and abundance of aberrant DNA methylation. Blood. 2009;114(16):3448-58. doi: 10.1182/blood-2009-01-200519.

4. Aggerholm A, Holm MS, Guldberg P, Olesen LH, Hokland P. Promoter hypermethylation of p15INK4B, HIC1, CDH1, and ER is frequent in myelodysplastic syndrome and predicts poor prognosis in early-stage patients. Eur J Haematol. 2006;76(1):23-32. doi: 10.1111/j.1600-0609.2005.00559.x.

5. Santini V, Melnick A, Maciejewski JP, Duprez E, Nervi C, Cocco L, et al. Epigenetics in focus: pathogenesis of myelodysplastic syndromes and the role of hypomethylating agents. Crit Rev Oncol Hematol. 2013;88(2):231-45. doi: 10.1016/j. critrevonc.2013.06.004.

6. Greenberg PL, Tuechler H, Schanz J, Sanz G, Garcia-Manero G, Solé F, et al. Revised international prognostic scoring system for myelodysplastic syndromes. Blood. 2012;120(12):2454-65. doi: 10.1182/ blood-2012-03-420489.

7. Cheson BD, Greenberg P, Bennett JM, Lowenberg B, Wijermans PW, Nimer SD, et al. Clinical application and proposal for modification of the International Working Group (IWG) response criteria in myelodysplasia. Blood. 2006;108(2):419-25. doi: 10.1182/blood-2005-10-4149.

8. Valent P, Horny HP, Bennett JM, Fonatsch C, Germing U, Greenberg P et al. Definitions and standards in the diagnosis and treatment of the myelodysplastic syndromes: consensus statements and report from a working conference. Leuk Res. 2007; 31(6):727-36. doi: 10.1016/j.leukres.2006.11.009.

9. de Witte T, Suciu S, Verhoef G, Labar B, Archimbaud E, Aul C, et al. Intensive chemotherapy followed by allogeneic or autologous stem cell transplantation for patients with myelodysplastic syn- dromes (MDSs) and acute myeloid leukemia following MDS. Blood. 2001;98(8):2326-31.

10. Silverman LR, Demakos EP, Peterson BL, Kornblith AB, Holland JC, Odchimar-Reissig R, et al. Randomized controlled trial of azacitidine in patients with the myelodysplastic syndrome: a study of the cancer and leukemia group B. J Clin Oncol. 2002; 20(10):2429-40. doi: 10.1200/JCO.2002.04.117.

11. Fenaux P, Mufti GJ, Hellström-Lindberg E, Santini V, Finelli C, Giagounidis A, et al. Efficacy of azacitidine compared with that of conventional care regimens in the treatment of higher-risk myelodysplastic syndromes: a randomised, open-label, phase III study. Lancet Oncol. 2009;10(3):223-32. doi: 10.1016/S1470-2045(09)70003-8.

12. Visser O, Trama A, Maynadi'e M, Stiller C, Marcos-Gragera R, De Angelis R, et al. RAREC-

ARE Working Group. Incidence, survival and prevalence of myeloid malignancies in Europe. Eur J Cancer. 2012;48(17):3257-66. doi: 10.1016/j. ejca.2012.05.024.

13. Dombret H, Seymour JF, Butrym A, Wierzbowska A, Selleslag D, Jang JH, et al. International phase 3 study of azacitidine vs conventional care regimens in older patients with newly diagnosed AML with $>30 \%$ blasts. Blood. 2015;126(3):291-9. doi: 10.1182/ blood-2015-01-621664. 